\title{
Influence du système de pâturage sur la croissance de jeunes bovins
}

\author{
D Dozias 1, D Micol 2 \\ IINRA, Domaine du Pin au Haras, 61310 Exmes ; INRA, Theix, 63122 St-Genès-Champanelle, France
}

L'effet de l'extensification de la conduite des prairies demande à être mieux caractérisé quant à la régularité de la production herbagère et des performances zootechniques permises. Dans les conditions herbagères du domaine du Pin au Haras (Normandie, France), nous décrivons ici la réponse, à deux modalités très écartées de gestion de la ressource herbagère, de jeunes bouvillons entre 6 et 13 mois d'âge. Ces animaux réalisent alors leur première saison de pâturage et comme ils possèdent peu de capacité à compenser, il importe de savoir si les différentes modalités de pâturage peuvent assurer régulièrement une croissance satisfaisante. 116 animaux normands ou normands-charolais ont été étudiés sur 4 années, de 1991 à 1994. Ils pesaient $180 \mathrm{~kg}$ à la mise à l'herbe, le 9 avril.

Le premier mode (I) correspond à une gestion intensive de la ressource herbagère. Les parcelles sont exploitées en rotation pendant 5 à 10 jours après 25 à 35 jours de repos. La fertilisation azotée (203 kg N/ha) assure des chargements instantanés élevés. L'utilisation de concentrés énergétiques (51 kg /animal) et de fourrages ( $70 \mathrm{~kg} \mathrm{MS} / \mathrm{animal}$ ) permet de tamponner l'effet des aléas climatiques sur l'offre alimentaire.

Le deuxième mode $(E)$ combine l'utilisation plus libérale des surfaces (pâturage continu au printemps, chargement instantané faible) à la suppression de la fertilisation azotée et à la diminution des intrants alimentaires. $19 \mathrm{~kg} / \mathrm{animal}$ de concentrés énergétiques et 70 $\mathrm{kg} \mathrm{MS/animal} \mathrm{de} \mathrm{fourrages} \mathrm{sont} \mathrm{distribués}$ durant les 3 semaines de transition après la mise à l'herbe.

De la mise à l'herbe au 15 mai, un animal du traitement I pâture 6,6 ares sur 2 parcelles, puis 21,7 ares sur 5 parcelles du 16 mai au 12 juillet. Dans le même temps, un bovin du traitement $E$ utilise 10,9 ares en pâturage continu.

Ensuite 6 parcelles offrant 28 ares par animal sont pâturées dans le traitement I en 2,5 cycles pendant que 3 parcelles représentant 48 ares par animal sont pâturées 1,5 fois dans le traitement $\mathrm{E}$.

Le 7 novembre, à la rentrée en stabulation à 12,5 mois d'âge, les animaux du traitement I pèsent $360 \mathrm{~kg} \pm 29$ contre $355 \mathrm{~kg} \pm 37$ pour ceux de la modalité $E$. Leur croissance respective ont été de $855 \mathrm{~g} / \mathrm{j}$ et de $845 \mathrm{~g} / \mathrm{j}$. Comme pour les poids, les croissances ne sont pas significativement différentes au seuil de $5 \%$. On peut toutefois observer dans le système plus extensif $(E)$ des performances plus irrégulières aussi bien intra-série qu'interséries (43 kg d'écart entre séries extrêmes à la rentrée en stabulation contre $34 \mathrm{~kg}$ pour le mode (). En moyenne, les croissances réalisées ne limitent pas le devenir de l'animal, quant à son âge et son poids d'abattage, mais un plus grand nombre d'animaux de la modalité $E$ devraient être destinés à des cycles longs. En effet, $47 \%$ des animaux du traitement $E$ pèsent moins de $350 \mathrm{~kg}$ à la rentrée en stabulation contre $37 \%$ dans le traitement I.

Au cours de la saison de pâturage, on observe pour la modalité $E$, un creux dans l'offre d'herbe, les repousses des parcelles fauchées arrivant environ 15 jours trop tard, ce qui explique le léger retard final de croissance des animaux de ce traitement.

Une meilleure régularité dans les poids finaux pourrait sans doute être obtenue dans le traitement $E$ en agissant sur un des trois facteurs de variation notamment l'utilisation de compléments alimentaires. 